\title{
EL LIDERAZGO EN EL CLIMA EMPRESARIAL MAO UNDERACTION EC DEL CANTÓN AMBATO DE LA PROVINCIA DE TUNGURAHUA
}

\section{LEADERSHIP IN BUSINESS CLIMATE MAO UNDERACTION EC OF CANTON AMBATO OF THE PROVINCE OF TUNGURAHUA}

Recebido: 30/10/2017 - Aprovado: 20/11/2017 - Publicado: 30/12/2017 Processo de Avaliação: Double Blind Review

\section{Jorge Vladimir Gonzales Reyes ${ }^{1}$}

Psicólogo Industrial

Universidad Técnica de Ambato

geoproduce1106@gmail.com

\section{Medardo Andrés Paredes Núñez}

Médico

Universidad Regional Autónoma de los Andes UNIANDES

andypa7@hotmail.es

\section{Rocío Núñez López}

Mg. Psicología educativa

Universidad Técnica de Ambato

rocionuniez@hotmail.com

\section{Verónica Paredes Núñez}

Mg. Administración de empresas turística y Hotelería

Universidad Autónoma de los Andes

vero_parredes@hotmail.com

\section{Isaac Israel Paredes Núñez}

Abogado

Universidad Católica del Ecuador

fatjoe16_16@hotmail.com

RESUMEN: El presente trabajo se basa en el liderazgo en el clima laboral de la empresa Mao Underaction EC del Cantón Ambato de la provincia de Tungurahua. El trabajo partió de la formulación de un objetivo general en torno al cual se traza cual es el liderazgo que caracteriza el trabajo diario dentro de las empresas y la relación existente entre el empleador y sus colaboradores, se prosigue con el marco teórico, que permite definir el concepto que debe marcar un lugar de trabajo armónico, para así poder alcanzar resultados de producción, con eficiencia y eficacia. Para lograrlo se realizó un análisis sobre la influencia del liderazgo en el clima laboral para determinar los efectos positivos en sus directivos y colaboradores. Dentro

\footnotetext{
${ }^{1}$ Autor para correspondência: Universidad Técnica de Ambato, Los Chasquis y Rio cutuchi, Av. de Los Chasquis, Ambato, Ecuador.
}

REMIPE- Revista de Micro e Pequenas Empresas e Empreendedorismo da Fatec Osasco 
de la metodología aplicada se eligió la modalidad de investigación aplicada de campo y como instrumento se empleó la encuesta, la población objeto de estudio seleccionada fue 50 personas de la empresa escogida como campo de estudio estuvo constituida por empleador y trabajadores. La encuesta estuvo estructurada así: 10 preguntas enfocadas hacia el concepto de Salario, definición del Ambiente Laboral, precisión de los Procesos, medición del nivel de Desempeño y Satisfacción Laboral, Entorno Físico, Relaciones Interpersonales, Valores Organizacionales, Normas y Autorrealización. Una vez evaluados los Resultados quedó demostrado que la mayoría de la población considera objeto de estudio expreso que la empresa no mantiene un liderazgo acorde a las expectativas de los trabajadores, lo cual influye de manera negativa en el rendimiento y eficiencia de la población trabajadora.

Palabras claves: liderazgo; calidad; clima laboral; eficacia.

ABSTRACT: The present work is based on the leadership in the company's work environment, Mao Underaction EC of Cantón Ambato of the province of Tungurahua. The work started from the formulation of a general objective around, which the leadership that characterizes the daily work within the companies and the relationship between the employer and his collaborators is traced, the theoretical framework is continued, which allows defining. The concept that should mark a harmonious workplace, in order to achieve production results, with efficiency and effectiveness. To achieve this, an analysis was made on the influence of leadership in the labor environment to determine the positive effects on its managers and employees. Within the applied methodology was chosen the applied research modality of field and as instrument was used the survey, the population object that was the purpose of study selected, it was 50 people of the company chosen as field of study and was constituted by employer and employees. The survey was structured as follow: 10 questions focused on the concept of salary, definition of the work environment, precision of the processes, measurement of the level of performance and work satisfaction, physical environment, interpersonal relationships, organizational values, procedures and self-fulfillment. Once the results were evaluated, it was demonstrated that the majority of the population considers the object of an express study that the company does not maintain leadership according to the expectations of the workers, which negatively influences the performance and efficiency of the working population.

Key words: leadership, quality; climate; labor; efficiency.

REMIPE- Revista de Micro e Pequenas Empresas e Empreendedorismo da Fatec Osasco 


\section{INTRODUCCIÓN}

En la actualidad las organizaciones deben ajustarse a las necesidades de sus trabajadores, de sus clientes y de su entorno social que cada vez es más competitivo y demanda una mayor presión en cuanto al cumplimiento de metas y objetivos empresariales, estas necesidades obligan a las organizaciones a estudiar e investigar qué tipo de liderazgo es el más adecuado para el desarrollo de las mismas, siendo capaces de adaptarse a los cambios internos y externos que la sociedad ejerce.

Este artículo tiene como finalidad exponer el estudio en torno al cual se traza cual es el liderazgo que caracteriza la calidad de trabajo diario dentro de las empresas y la relación existente entre el empleador y sus colaboradores. El Factor liderazgo potencia el compromiso de sus subordinados con la visión y misión propuesta por la empresa, lo cual hoy día trasciende fronteras, concepto el cual es muy necesario de tener presente en estos tiempos difíciles, ya que la esencia de este concepto trasmite a sus seguidores sensaciones de empoderamiento, delega autoridad y forma nuevos líderes, hace énfasis en la resolución de problemas y un buen uso de razonamiento. En consecución se logra la promoción del interés e incrementa la confianza de los colaboradores, gradualmente los sitúa en un estado de compromiso y desarrollo de sí mismos.

Actualmente la importancia del clima laboral es muy valorada por las organizaciones, las cuales afirman que es un factor fundamental para el desarrollo personal y profesional de los colaboradores, ya que posibilita a los directivos obtener una visión a corto y largo plazo de la organización. Sin duda, que el liderazgo es un factor que permite identificar la realidad cambiante de su entorno, ya que permite identificar las necesidades de todos y cada uno de los miembros que conforman la empresa para de esta forma proponer posibles soluciones para el mejoramiento del clima laboral dentro y fuera de la organización.

Es por ello, que el presente trabajo de investigación presenta aun marco teórico que sustenta los conceptos, definiciones y teorías que sustentan la importancia del liderazgo en el clima organizacional dentro de cualquier empresa, ya que se ha identificado que el liderazgo transformacional influye de forma positiva en el clima laboral brindando bienestar a los trabajadores, porque generan ambientes de trabajo motivantes, que les permite a los empleados crecer y desarrollarse tanto en lo personal como profesionalmente. 


\section{Objetivo General}

Determinar la influencia del liderazgo dentro del clima organizacional de una empresa de la población del área Técnica y Administrativa de la Empresa Mao Underaction EC del Cantón Ambato de la provincia de Tungurahua.

\section{ESTADO DEL ARTE}

\subsection{CLIMA LABORAL}

Por clima laboral se entiende el conjunto de cualidades, atributos o propiedades relativamente permanentes de un ambiente de trabajo concreto que son percibidas, sentidas o experimentadas por las personas que componen la organización y que influyen sobre su conducta. Uno de los elementos de estudios más considerados que influyen en el clima laboral es el concepto de liderazgo.

El clima organizacional ha ido evolucionando su definición a lo largo del tiempo, ya que este tiene una prolongada historia en las ramas de la psicología, industrial y comportamiento organizacional (Guillen \& Guil, 2000). Este tema llega a conceptualizarse en 1968 cuando se determina que el clima organizacional es medible, estudiándolo como una función de comportamientos que los trabajadores tienen según sus valores para con el mismo y según sus necesidades de trabajo (Schneider, 1990).

El liderazgo es un tema de gran importancia en las organizaciones, tal como se ha mencionado, el principal elemento con que cuenta una empresa o institución es el factor humano, sin embargo, existe una persona responsable de dirigir los esfuerzos y recursos que forman parte de ésta. Es por ello que el liderazgo toma un papel fundamental pues debe ser efectivo para lograr el propósito de influir en el personal y de esta manera se logren los objetivos planteados.

En la Teoría del liderazgo transformacional y transaccional de Bass y AvolioBass y Avolio (1994) los cuales definen que el liderazgo transformacional está enfocado en elevar el interés de los seguidores, el desarrollo de la autoconciencia y aceptación de la misión organizacional y el ir más allá de sus autointereses por el interés del bienestar del grupo. esperado.

El líder transformacional inspira a los seguidores para alcanzar más de lo

Involucra una fuerte identificación entre el personal con el líder.

Integra una visión compartida del futuro, logrando ir más allá del intercambio de intereses sobre premios contingentes.

REMIPE- Revista de Micro e Pequenas Empresas e Empreendedorismo da Fatec Osasco 
El efecto transformador de dichos líderes se observa, tanto en las organizaciones, grupos, como individuos.

En este sentido y de acuerdo con la forma de actuar del líder transformacional, según Mendoza (2008), el liderazgo tiene una relación importante con tres aspectos: la satisfacción de los individuos y el grupo, el esfuerzo extra en su desempeño y la efectividad.

Existe diversidad de perspectivas sobre el tema, a continuación se mencionarán algunas aportaciones de acuerdo con la propuesta de Castro y Lupano (2007), la primera es la denominada Teoría relacional del liderazgo de Graen y Uhl-Bien en 1995, la cual se basa en el análisis de las interacciones que se constituyen entre líder y seguidor. Dicha teoría hace énfasis en las relaciones de alta calidad representadas por la confianza y el respeto mutuo, lo cual genera resultados positivos por parte del líder, en oposición al establecimiento de relaciones de baja calidad que son caracterizadas por un vínculo meramente contractual atravesado por obligaciones mutuas. Igualmente en 2006 Bass y Riggio (citados en Castro y Lupano, 2007) afirman que el modelo de liderazgo transformacional ha evolucionado hacia un modelo denominado Liderazgo de Rango Completo (Full Range Leadership), el cual contempla además de las dimensiones de liderazgo transformacional y transaccional, una última dimensión denominada liderazgo Laissez Faire. De las definiciones mencionadas anteriormente expresadas por distintos autores, se infiere que el liderazgo es un proceso por medio del cual un individuo ejerce influencia sobre otros para lograr objetivos.

\subsection{RELACIÓN LIDERAZGO-CLIMA ORGANIZACIONAL}

El liderazgo es un concepto altamente complejo, cuyo axioma y cuyo alcance mantienen una íntima vinculación con los acercamientos teóricos contemporáneas, los mismos provienen de las corrientes epistemológicas que han inscrito los diferentes cursos de la historia. Con esta base el liderazgo debe ser estudiado desde su historia para que pueda ser entendido mejor (Friedman, 2010). Por tal razón se puede confirmar que el liderazgo es un concepto tanto cultural y social como histórico que envuelve una comprensión de las organizaciones en algunos contextos (Contreras, 2008).

"El liderazgo es la capacidad de influir en un grupo para que se logren las metas" (Gómez, 2002). Para Davis y Newstrom (2003) el Liderazgo es el proceso de lograr influir sobre los demás con trabajo en equipo, con el propósito de que trabajen con entusiasmo en el logro de sus objetivos. Payeras (2004) lo explica como un grupo de formas de comportamiento que el líder usa como herramienta para influir sobre el actuar de los individuos y equipos. Esta influencia se desarrolla a través de una visión sustentada por los valores que la apoyan, 
generando en el individuo la incorporación a su propio comportamiento (Hellriegel \& Slocum, 2004).

El chispazo básico del liderazgo descansa en que las personas son proclives a seguir a aquellos que pueden participar positivamente en el alcance de sus metas (Koontz \& O’Donell, 2001). En este sentido el rol del líder es lograr que la organización genere relaciones que permitan obtener los resultados esperados, ya que los líderes promueven esfuerzos para que su trabajo se cristalice en la creación de nuevas oportunidades que permitan compartir y aprender lo aprendido, llegando a transformar el contexto (Fullan, 2002).

La efectividad del líder está marcada por su habilidad para hacer que las organizaciones logren las metas planteadas, considerando su habilidad para influir sobre el proceder de los demás (Robbins \& Judge, 2009). Por eso el liderazgo para obtener un resultado positivo debe desarrollarse "con una visión integrada por el líder, el talento humano y la organización, dirigida a la búsqueda de la calidad y excelencia organizacional" (Cruz \& Salanova, 2011).

Desde esta perspectiva, para Perdomo \& Prieto (2009), "el rol del liderazgo involucra promover el crecimiento y la participación personal, estimular la creatividad, y ampliar habilidades en todos los miembros de la organización". Para que esto sea así, el líder tiene que poseer la capacidad social y cognitiva para aportar, crear y desarrollar equipos altamente efectivos (Uribe, Molina, Contreras, Barbosa, \& Espinosa, 2013). Como consecuencia de lo anteriormente expuesto se concebirán, al interior de las organizaciones procesos de liderazgo flexibles que acrecienten las capacidades para analizar e interpretar el entorno y favorezcan al cambio organizacional para responder efectivamente a las amenazas y oportunidades (Robles, Contreras, Barbosa, \& Juárez, 2013).

Raineri (2006) en su estudio señaló que existe correlación entre las variables liderazgo y el clima organizacional. De la misma manera (Ponce, et. al, 2014) en su investigación dio por comprobado que la percepción de los trabajadores sobre el clima organizacional es fuertemente influenciada por el estilo de liderazgo, y que los resultados organizacionales son afectados a medida que la percepción del clima organizacional es favorable o desfavorable.

De la Garza, Ruiz, y Hernández (2013) encontraron en su investigación que existe correlación entre la motivación que el líder proyecta y el clima organizacional satisfactorio, demostrando que cuando un líder incentiva a mejorar la calidad del desempeño, contribuye a que los empleados sientan que su trabajo contribuye a mejorar la calidad de la organización. De igual manera Pons y Ramos (2012) en su estudio concluyen que los resultados obtenidos REMIPE- Revista de Micro e Pequenas Empresas e Empreendedorismo da Fatec Osasco 
refuerzan la relación que tiene el liderazgo empleado en una organización y la percepción de los trabajadores sobre el clima organizacional.

Por otra parte, González, Figueroa y González (2014) en su investigación demostraron que el liderazgo influye sobre el clima organizacional, determinando asimismo que el liderazgo influye también sobre las otras dimensiones del clima organizacional, en especial y con mayor énfasis en la motivación. A través del instrumento Work Evironment Scale (WES), Lam, Cisneros, Bravo, Carrillo y Bustamante (2013) demostraron que existe una fuerte relación entre el liderazgo y el clima organizacional, confirmando también que existe correlación entre el clima organizacional y las relaciones interpersonales.

En función del marco teórico estudiado se procedió a seleccionar el marco metodológico que sustentaría el estudio de la presente investigación.

\section{METODOLOGÍA}

La investigación adopta dos métodos: cuantitativo y cualitativo para así poder investigar de qué forma incide el Liderazgo en el Clima Organizacional de la empresa

La modalidad básica fue en primer término, de carácter bibliográfica, por lo cual se desarrolló una investigación documentada en libros, revistas, tesis, y artículos en los que se observaron sistemáticamente las realidades. Esta investigación será apoyada por un estudio de campo, aplicando el instrumento de la encuesta, para analizar los resultados a través de tabulaciones, análisis e interpretaciones de los datos obtenidos.

\section{ANÁLISIS E INTERPRETACIÓN}

La mayoría de la población entrevistada manifiesta que no existe un buen liderazgo en la empresa, mientras que un $24 \%$ afirma que existe parcialmente, y un $4 \%$ considera que sí, existe un buen liderazgo en la empresa.

Tabla 1 - ¿Piensa que existe un buen liderazgo en la empresa?

\begin{tabular}{|l|r|r|}
\hline ALTERNATIVA & FRECUENCIA & PORCENTAJE \\
\hline SI & 2 & $4,00 \%$ \\
\hline PARCIALMENTE & 12 & $24,00 \%$ \\
\hline NO & 36 & $72,00 \%$ \\
\hline TOTAL & 50 & $100,00 \%$ \\
\hline
\end{tabular}

Elaborado por el autor, 2017.

REMIPE- Revista de Micro e Pequenas Empresas e Empreendedorismo da Fatec Osasco 
Figura 1 Liderazgo de Autoridades

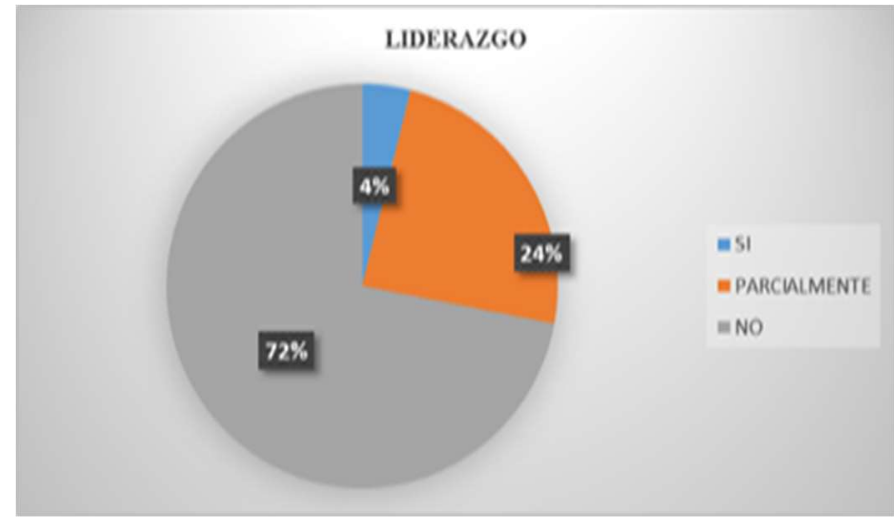

Elaborado por los autores, 2017.

Del análisis hecho se observa que un 33\% afirma que parcialmente el liderazgo de las autoridades aporta de manera efectiva al clima laboral, mientras que un $17 \%$ de la población objeto de estudio considera que sí, aporta. El liderazgo en la organización está aportando las decisiones y actividades que los colaboradores deben seguir, deben darse por parte de jefes departamentales, gerencia, trabajadores con cargo de responsabilidades importantes, siendo importante ya que debe ser correctamente aprendido desde los altos a los bajos mandos.

Tabla 2 - ¿Cree que el liderazgo de las autoridades aporta de manera efectiva al clima organizacional?

\begin{tabular}{|l|r|r|}
\hline ALTERNATIVA & FRECUENCIA & PORCENTAJE \\
\hline SI & 17 & $34,00 \%$ \\
\hline PARCIALMENTE & 33 & $66,00 \%$ \\
\hline NO & 0 & $0,00 \%$ \\
\hline TOTAL & 50 & $100,00 \%$ \\
\hline
\end{tabular}

Elaborado por los autores, 2017. 
Figura 2 Liderazgo

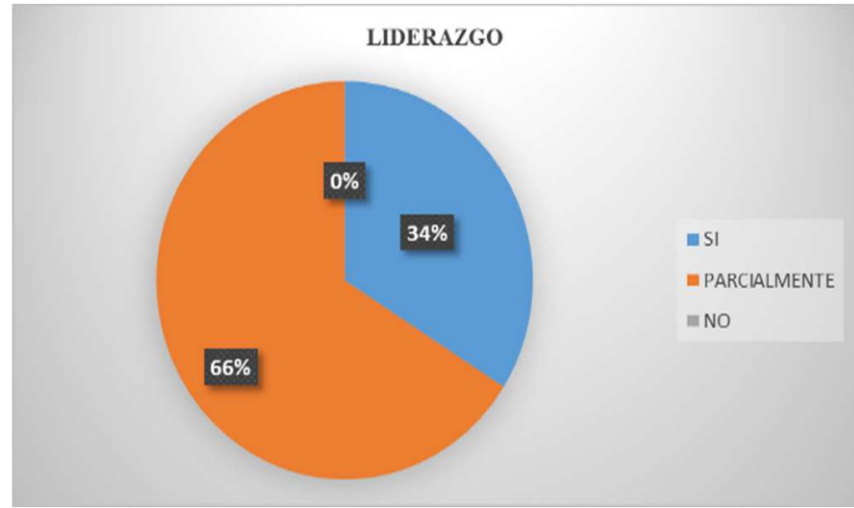

Elaborado por los autores, 2017.

E1 50\% de las personas entrevistadas manifiesta que no existe compromiso de trabajo por parte de las autoridades de la empresa, mientras que el 10\% indica que el compromiso por parte de los altos mandos que ese compromiso se da de manera parcial, mientras el $40 \%$ menciona que sí, existe un compromiso de trabajo por parte de las autoridades de la empresa

Tabla 3 - ¿Cree que existe compromiso de trabajo por parte de las autoridades de la empresa?

\begin{tabular}{|l|r|r|}
\hline ALTERNATIVA & FRECUENCIA & PORCENTAJE \\
\hline SI & 20 & $40,00 \%$ \\
\hline PARCIALMENTE & 5 & $10,00 \%$ \\
\hline NO & 25 & $50,00 \%$ \\
\hline TOTAL & 50 & $100,00 \%$ \\
\hline
\end{tabular}

Elaborado por los autores, 2017.

Figura 3 - Compromiso de las autoridades

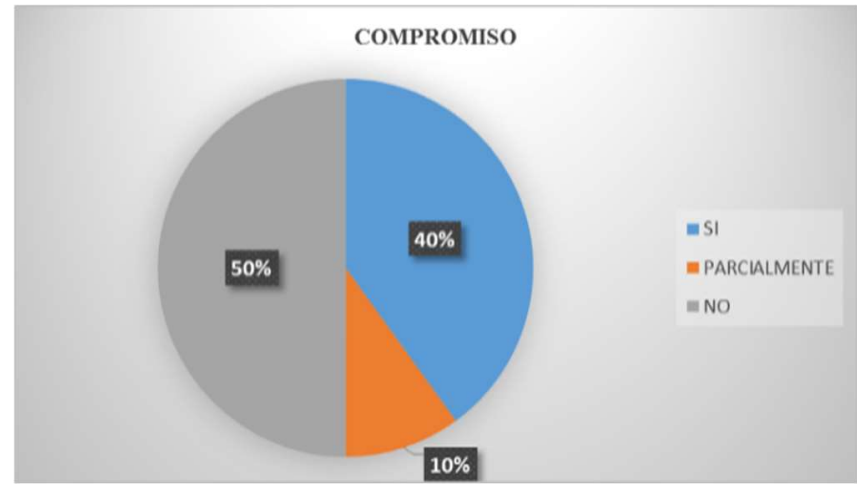

Elaborado por los autores, 2017. 
De la población encuestada un $68 \%$ considera que su salario es parcialmente justo en cuanto a las actividades que realiza en la empresa, mientras que un $28 \%$ considera la remuneración que percibe es justa y solamente un 4\% indica que considera injusto el salario que recibe.

Tabla 4 - ¿Considera que su salario es justo en cuanto a las actividades que realiza en la empresa?

\begin{tabular}{|l|r|r|}
\hline ALTERNATIVA & FRECUENCIA & PORCENTAJE \\
\hline SI & 14 & $28,00 \%$ \\
\hline PARCIALMENTE & 34 & $68,00 \%$ \\
\hline NO & 2 & $4,00 \%$ \\
\hline TOTAL & 50 & $100,00 \%$ \\
\hline
\end{tabular}

Elaborado por los autores, 2017.

Figura 4 - Salario Justo

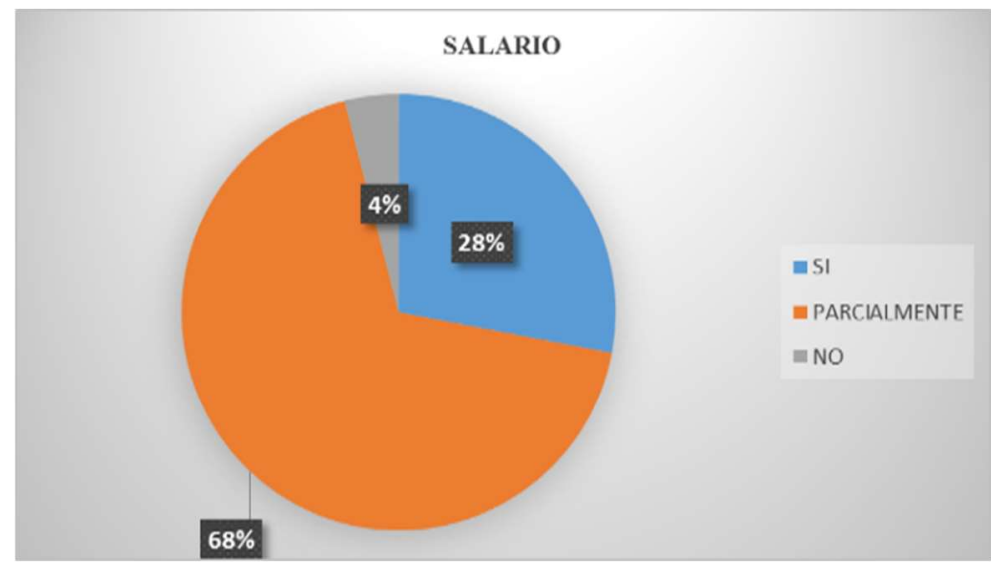

Elaborado por los autores, 2017.

Un $72 \%$ de los encuestados indica que no existe una buena comunicación entre compañeros al momento de desempeñar su trabajo, mientras que un $26 \%$ afirma que existe parcialmente una buena comunicación entre compañeros, y solo un $2 \%$ respondió que existe una buena comunicación entre compañeros dentro de la empresa. 
Tabla 5 - ¿Cree que existe una buena comunicación entre compañeros al momento de desempeñar su trabajo?

\begin{tabular}{|l|r|r|}
\hline ALTERNATIVA & FRECUENCIA & PORCENTAJE \\
\hline SI & 1 & $2,00 \%$ \\
\hline PARCIALMENTE & 13 & $26,00 \%$ \\
\hline NO & 36 & $72,00 \%$ \\
\hline TOTAL & 50 & $100,00 \%$ \\
\hline
\end{tabular}

Elaborado por los autores, 2017.

Figura 5 - Comunicación

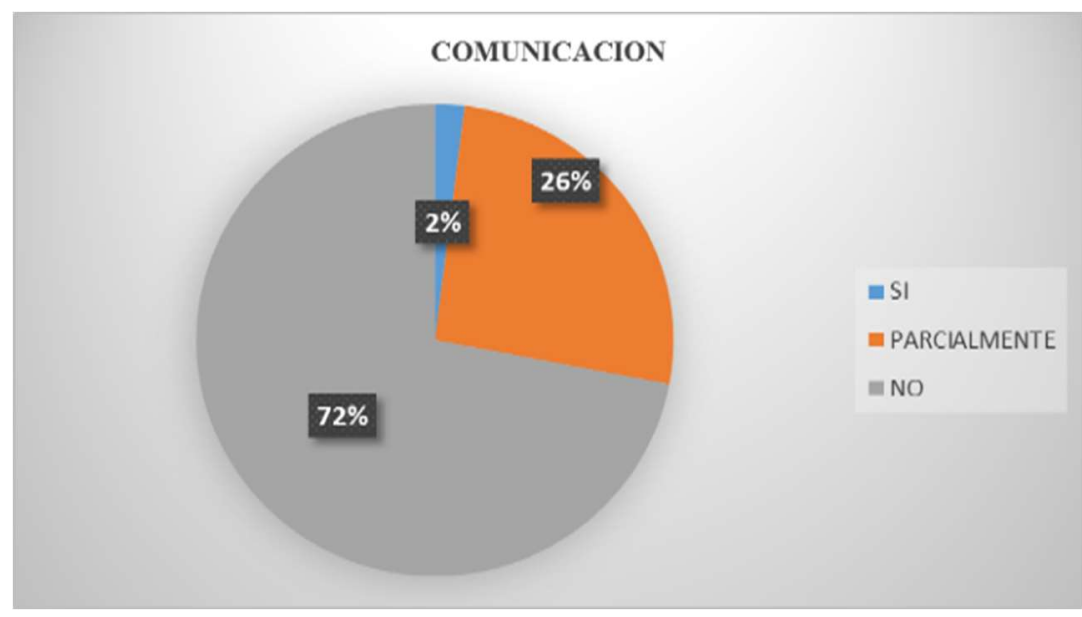

Elaborado por los autores, 2017.

El 50\% de los encuestados responde que efectivamente su área de trabajo le permite realizar sus actividades laborales con comodidad, mientras que el 40\% manifiesta que esta comodidad se da de manera parcial, mientras que un 10\% menciona que está satisfecho con la comodidad que tiene en su área laboral.

El área de trabajo es el lugar en donde desarrollan sus funciones los colaboradores de una organización, por tanto, debe cumplir correctamente con los estándares mínimos establecidos $\mathrm{y}$ debe ser cómoda, en todo momento debe evitar que se desarrollen enfermedades ocupacionales y de trabajo. 
Tabla 6 - ¿Su área de trabajo le permite realizar sus actividades con comodidad?

\begin{tabular}{|l|r|r|}
\hline ALTERNATIVA & FRECUENCIA & PORCENTAJE \\
\hline SI & 5 & $10,00 \%$ \\
\hline PARCIALMENTE & 20 & $40,00 \%$ \\
\hline NO & 25 & $50,00 \%$ \\
\hline TOTAL & 50 & $100,00 \%$ \\
\hline
\end{tabular}

Elaborado por los autores, 2017.

Figura 6 - Área de Trabajo

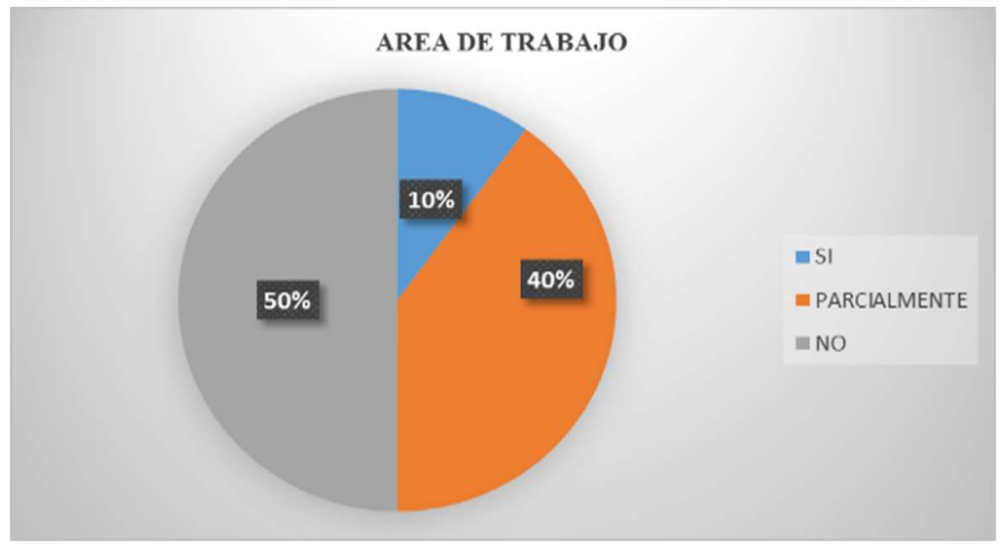

Elaborado por los autores, 2017.

De la población encuestada un 56\% manifiesta que sí, existen discusiones entre compañeros dentro de la empresa, mientras que un 30\% expresa que sí, existen discusiones pero de manera parcial y un 14\% manifiesta que no existen discusiones entre los compañeros de la empresa.

Tabla 7 - ¿Existen discusiones entre compañeros dentro de la empresa?

\begin{tabular}{|l|r|r|}
\hline ALTERNATIVA & FRECUENCIA & PORCENTAJE \\
\hline SI & 28 & $56,00 \%$ \\
\hline PARCIALMENTE & 15 & $30,00 \%$ \\
\hline NO & 7 & $14,00 \%$ \\
\hline TOTAL & 50 & $100,00 \%$ \\
\hline
\end{tabular}

Elaborado por los autores, 2017. 
Figura 7 - Compañerismo

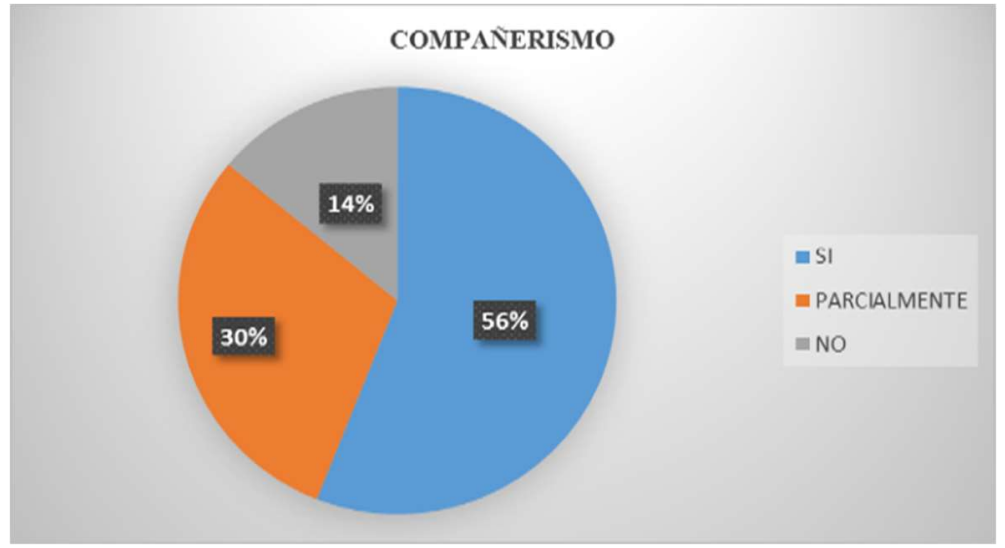

Elaborado por los autores, 2017.

De la población encuestada un 56\% expresa que los empleados no son capacitados sobre valores organizacionales, mientras que un $42 \%$ afirma que esta capacitación se da pero de manera parcial y un $2 \%$ manifiesta que sí, son capacitados los empleados sobre valores organizacionales

Tabla 8 - ¿Los empleados son capacitados sobre valores

\begin{tabular}{|l|r|r|}
\hline \multicolumn{3}{|c}{ organizacionales? } \\
\hline ALTERNATIVA & FRECUENCIA & PORCENTAJE \\
\hline SI & 1 & $2,00 \%$ \\
\hline PARCIALMENTE & 21 & $42,00 \%$ \\
\hline NO & 28 & $56,00 \%$ \\
\hline TOTAL & 50 & $100,00 \%$ \\
\hline
\end{tabular}

Elaborado por los autores, 2017.

Figura 8 - Valores Organizacionales

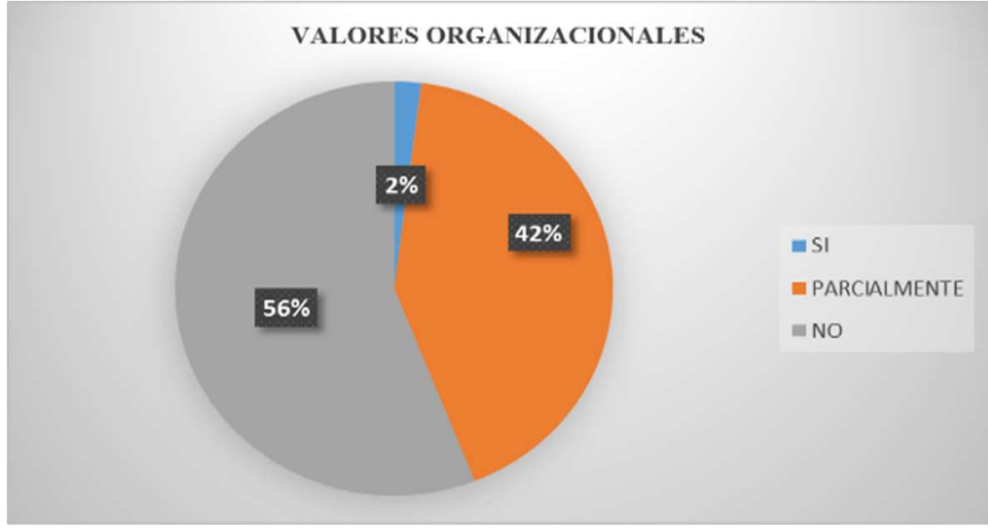

Elaborado por los autores, 2017. 
Tabla 9 - ¿Considera que su nivel de autoestima varía según el trabajo que realice en la empresa?

\begin{tabular}{|l|r|r|}
\hline ALTERNATIVA & FRECUENCIA & PORCENTAJE \\
\hline SI & 25 & $50,00 \%$ \\
\hline PARCIALMENTE & 9 & $18,00 \%$ \\
\hline NO & 16 & $32,00 \%$ \\
\hline TOTAL & 50 & $100,00 \%$ \\
\hline
\end{tabular}

Elaborado por los autores, 2017.

La mitad de la población encuestada menciona que su nivel de autoestima, sí, varía según el trabajo que realice en la empresa, un 18\% menciona que su autoestima se ve influida parcialmente según el trabajo que realice en la empresa, mientras que un 32\% manifiesta que en nada influye su nivel de autoestima en las actividades laborales que realiza.

Figura 9 - Autoestima

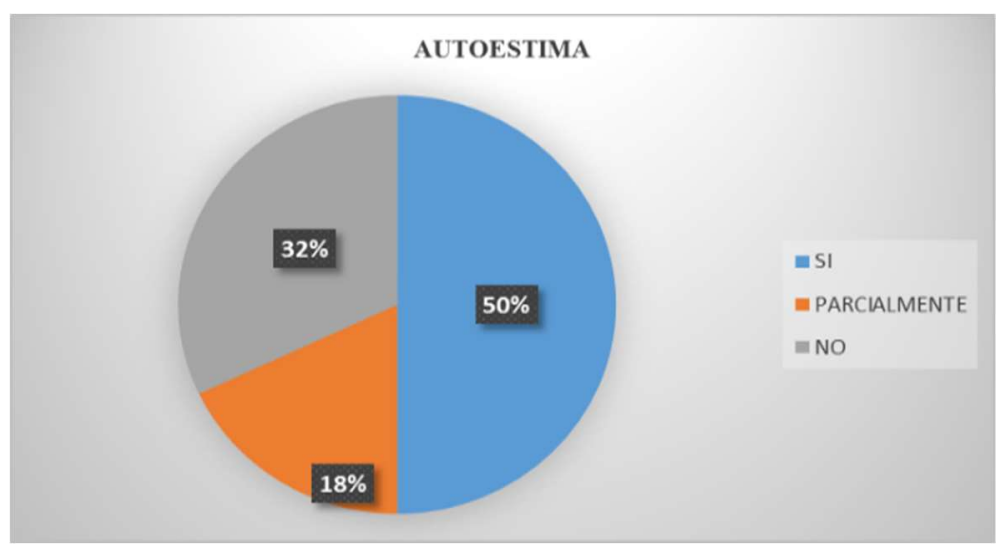

Elaborado por los autores, 2017.

El 52\% de la población señala que no existe un buen clima laboral, mientras que un $38 \%$ considera que, sí, existe un buen clima organizacional y un $10 \%$ manifiesta que existe de manera parcial en la empresa un buen clima organizacional. 
Tabla 10 - ¿Considera que en la empresa existe un buen clima organizacional?

\begin{tabular}{|l|r|r|}
\hline ALTERNATIVA & FRECUENCIA & PORCENTAJE \\
\hline SI & 19 & $38,00 \%$ \\
\hline PARCIALMENTE & 5 & $10,00 \%$ \\
\hline NO & 26 & $52,00 \%$ \\
\hline TOTAL & 50 & $100,00 \%$ \\
\hline
\end{tabular}

Elaborado por los autores, 2017.

Figura 10 - Clima Organizacional

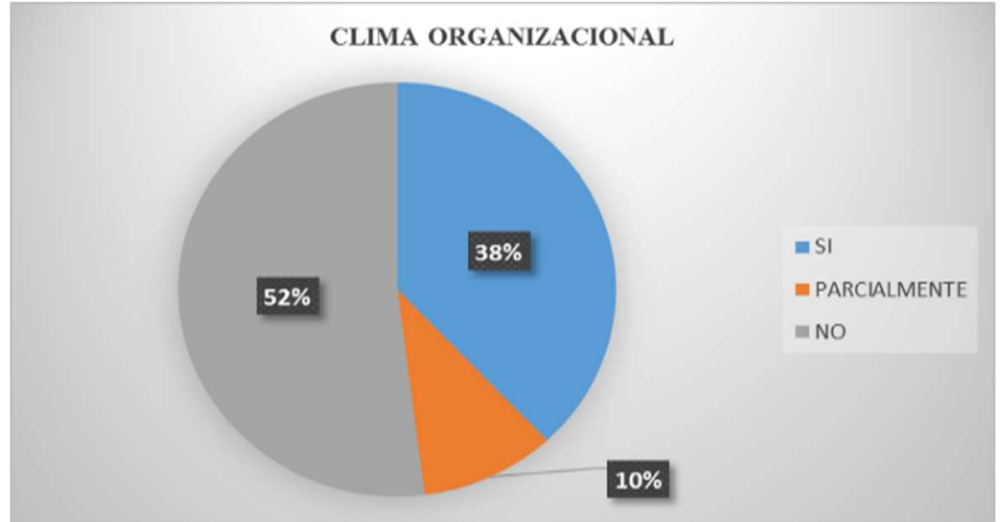

Elaborado por los autores, 2017.

\section{DISCUSIÓN DE RESULTADOS}

Los resultados de la encuesta aplicada a 50 personas de la empresa objeto de estudio permiten determinar que dentro del clima organizacional existe una ausencia de liderazgo por parte de quienes tienen la responsabilidad de dirigir la empresa, aun así, los empleados consideran que es muy importante que ese liderazgo sea de manera efectiva y asertiva, lo cual influiría positivamente en el rendimiento empresarial. Se pudo identificar que existe una deficiente comunicación, convirtiéndose en un problema relevante para la organización, generando dudas y desconfianza entre compañeros. Los empleados perciben que los responsables de la empresa deben fortalecer su nivel de compromiso con la misma, para obtener mejores resultados.

Esta situación desencadena conflictos laborales como la desmotivación de los empleados, afectando directamente a la productividad, disminuyendo su rendimiento, provocando malestar y confusión entre los trabajadores al momento de entender cuáles son las actividades que tienen que realizar en la jornada laboral. 
Esto lleva a que cada empleado entienda las instrucciones a su manera, afectando el resultado de las metas y objetivos propuestos por la organización. Positivamente se observa que los resultados arrojan que en su mayoría los empleados se encuentran parcialmente satisfechos con la remuneración salarial que reciben

En el estudio realizado se destaca por la importancia del rol que desempeña el trabajador dentro de la empresa como elemento que influye en su autoestima, ya que esta varía en proporción al papel que le corresponde desempeñar dentro del clima organizacional que pertenece. La actualización y capacitación del personal sobre los valores organizacionales dentro de la empresa son muy determinantes para crear sentido de pertenencia que potencie en el empleado vinculaciones afectivas y efectivas con la organización a la cual pertenece. Una empresa no debe soslayar este elemento como factor de desarrollo humano. En el análisis del factor clima organizacional se evidencia que se necesita favorecer condiciones más idóneas que permitan al trabajador sentirse a gusto dentro de su ambiente de trabajo, ya que un alto porcentaje de empleados que se encuentran insatisfechos y otros que expresan dudas y confusión con respecto a este factor de mucha importancia dentro y fuera de la organización.

\section{CONCLUSIONES}

Se puede mencionar que el liderazgo de las autoridades de una empresa es sumamente importante ya que constituye el ejemplo que los colaboradores deben seguir para poder cumplir los objetivos de la misma. La responsabilidad de las autoridades de la empresa debe ser el mejor para obtener los resultados más adecuados, es decir, que de esto parte la predisposición que tengan los empleados y colaboradores de la organización para poder seguir reglas y conseguir los objetivos deseados y propuestos en la planificación estratégica empresarial.

En el estudio realizado, los resultados arrojaron que la comunicación se maneja de forma inadecuada, lo cual afecta al clima organizacional y las relaciones interpersonales entre empleados, desmotivando a los mismos de manera directa, lo cual incide negativamente en su rendimiento laboral, disminuyendo su capacidad productiva y generando desconfianza entre las autoridades y los colaboradores del área técnica y administrativa de la empresa

Se concluye que el compromiso de trabajo por parte de las autoridades no satisface las necesidades de los empleados, creando un ambiente de dudas y desconfianza. Se debería organizar integraciones para fomentar la participación en la toma de decisiones en beneficio 
de la organización, promoviendo la motivación y la autoestima, tanto de los directivos como de los trabajadores de la empresa.

Se determina que el liderazgo dentro del clima organizacional de la empresa de la población del área Técnica y Administrativa de la Empresa Mao Underaction EC del Cantón Ambato de la provincia de Tungurahua no cumple con los lineamientos que se necesita para que se desempeñe con efectividad. El estudio realizado permitió identificar los puntos más débiles, los cuales son indispensables de fortalecer dentro de la organización, para así crear estrategias de acción que permitan obtener resultados en función de los criterios que se establecen como válidos en una Relación liderazgo-clima organizacional.

\section{BIBLIOGRAFÍA}

Alatorre, A. V. (2013). Interdependencia entre el liderazgo transformacional, cultura organizacional y cambio educativo: una reflexión. Revista Iberoamericana sobre Calidad, Eficacia y Cambio en Educacion (2013)

Antonakis, \& House. (2002). Liderazgo Transformacional y Carismatico. Oxford: Elsevier Science

Bass, R., \& Riggio, B. (2006). Liderazgo Transformacional. New Jersey: Lawrence Erlbaum Associates.

Blanch Rivas, J. M. (2003). Teoría de las Relaciones Laborales. Fundamentos. Barcelona..

Brunet, L. (2004). El Clima de Trabajo en la Organizaciones: Definicion Diagnostica y Consecuencias. Mexico

Burns, 1978 El liderazgo transformacional pag. 105

Burns, J. M. (1978). Leadership. Nueva York: Harper y Row.

Camacho, P. (2012). La evolucion del liderazgo en la actualidad . Colombia : Diplomado Gestionn para el desarrollo humano en la organizacion .

Cruz, O. (2011). Liderazgo Transformacional y su relacion con el Capital Psicologico Positivo Grupal . Madrid.

Curtis, E., \& O'Conell, R. (2011). Essencial Leadershipskills for motivating and developing staff, Nursing Management.

Curtis, E., \& O'Conell, R. 2011 . Essential leadership skills for motivating and developing staff. Curtis E(1), O'Connell R. Author information:

Clifford, R. A. 2014 . Publications Present Professor Nick Clifford - King's College

Denison, R. (1991). Cultura Corporativa y Productividad Organizacional. Colombia.

REMIPE- Revista de Micro e Pequenas Empresas e Empreendedorismo da Fatec Osasco 
Feinberg, J. (2005). El papel dentro del grupo de acuerdo en la comprension del liderazgo transformacional . Revista de organizacion y ocupacion psicologica .

Goleman. (2000). Leadership that gets results Hardvare Business Review.

Gonzalez, \& Figueroa. (2014). Influencia del Liderazgo sobre el Clima Organizacional en Pymes, caso catering gourmet de Durango. Mexico: Revista Internacional Administracion y Finanzas.

Guillen and Guil (2000) C. Guillen, R. Guil. Psicología del trabajo para las relaciones laborales.

Koontz H. y Heinz, W. (2003). Administracion: Una perspectiva Global. México.

Likert Rensis (1903-1981) Introducción al liderazgo organizacional, teoría y metodología

Pons F.J \& Ramos J. (2012). Influencia de los Estilos de Liderazgo y las Prácticas de Gestión de RRHH sobre el Clima Organizacional de Innovación. Revista Scielo. Obtenido de http://scielo.isciii.es/scielo.php?pid=S1576-

$59622012000200002 \&$ script $=$ sci_arttext\&tlng=entarget=blank

Robles et al., 2013. V. Robles, F. Contreras, D. Barbosa, F. Júarez. Liderazgo en directivos colombiano vs. mexicanos: Un estudio compartivo. Investigación\&Desarrollo, 21 (2013), pp. 395-418.

Raineri, 2006. Estilos de dirección como determinante del clima laboral en Chile. ABANTE, 9 (1) (2006), pp. 3-33. 